\title{
Efectos colaterales positivos de la pandemia
}

\author{
Dr. Walter Reyes Caorsi
}

"No es la más fuerte de las especies la que sobrevive, tampoco
es la más inteligente la que sobrevive. Es aquella que se adapta
mejor al cambio".
Charles Darwin (1809-1882).

\section{Introducción}

La pandemia originada por el coronavirus tendrá consecuencias importantes sobre la humanidad. Mas allá de las pérdidas humanas que, como nunca en la historia son contabilizadas diariamente, los efectos a mediano y largo plazo en las relaciones sociales y personales, el trabajo y el empleo, la economía (la macro y la personal), la política, los sistemas de salud, la cultura, el turismo y otros múltiples aspectos de nuestro llamado estilo de vida, marcarán un antes y un después que solo la perspectiva del tiempo permitirá valorar adecuadamente.

La mayoría de las consecuencias son percibidas a priori como negativas, y muchas seguramente lo serán para muchísimas personas en múltiples asuntos. Pero, aun entre aquellos que sufrirán menos esta crisis, existe la percepción de que deberemos modificar algunos hábitos para adaptarnos a la nueva realidad de la sociedad, y que quizás seremos menos libres.

Pero no todo será negativo. Nos referiremos a algunas consecuencias de esta circunstancia global, particularmente sobre nuestra actividad profesional en cardiología, que, de ser aprovechadas, deberán ser consideradas como efectos colaterales positivos. Simbolizan un cambio cultural en nuestra práctica, al cual deberemos adaptarnos. Seguramente no serán los únicos efectos de la pandemia sobre la medicina cardiovascular y muchas otras disciplinas.

\begin{abstract}
Tecnología
Uno de los primeros hechos a destacar durante esta epidemia es que la tecnología pasó a ser una herramienta muy importante en la vida de un mayor número de personas. Tecnología que, si bien estaba disponible, era utilizada en menor medida por un número más limitado de individuos, marcando, además, una diferencia sustancial con todas las otras epidemias que sufrió la humanidad, ya que permitió mantener el distanciamiento físico, sin aislamiento social. O incluso aumentando esa vinculación social y también familiar en algunos casos. Por supuesto que desde el punto de vista de las relaciones humanas, no son vínculos comparables, pero sin duda debemos reconocer que ha colaborado en hacer más tolerables las limitaciones.

\footnotetext{
"La tecnología es importante, pero lo único que realmente importa es lo que hacemos con ella". Muhammad Yunus, Premio Nobel de la Paz 2006.
}

\section{Telemedicina}

Telemedicina o telesalud, como algunos prefieren denominarla, es un término utilizado para referirse al diagnóstico o control del paciente a través de la tecnología. Se trata de un entorno asistencial y del cuidado de la salud potenciado por el uso de esa herramienta, que incluye visitas virtuales (e-consulta), monitoreo remoto de parámetros biológicos, o de dispositivos que los controlan (https://www. cchpca.org/ about/about-telehealth). Existen evidencias de los beneficios de su uso como complemento de la asistencia presencial tradicional, sin perjudicar una adecuada relación médico-paciente, y mejorando el compromiso del paciente en su propio control.

Esta forma de vínculo asistencial se potenció significativamente en muchos países del mundo aunque en una forma más limitada en el nuestro. Es un

Comisión Honoraria para la Salud Cardiovascular. Montevideo, Uruguay.

Correspondencia: Dr. Walter Reyes Caorsi. Correo electrónico: wreyes001@gmail.com

El autor declara no tener conflictos de intereses.

Recibido May 25, 2020; aceptado Jun 2, 2020. 


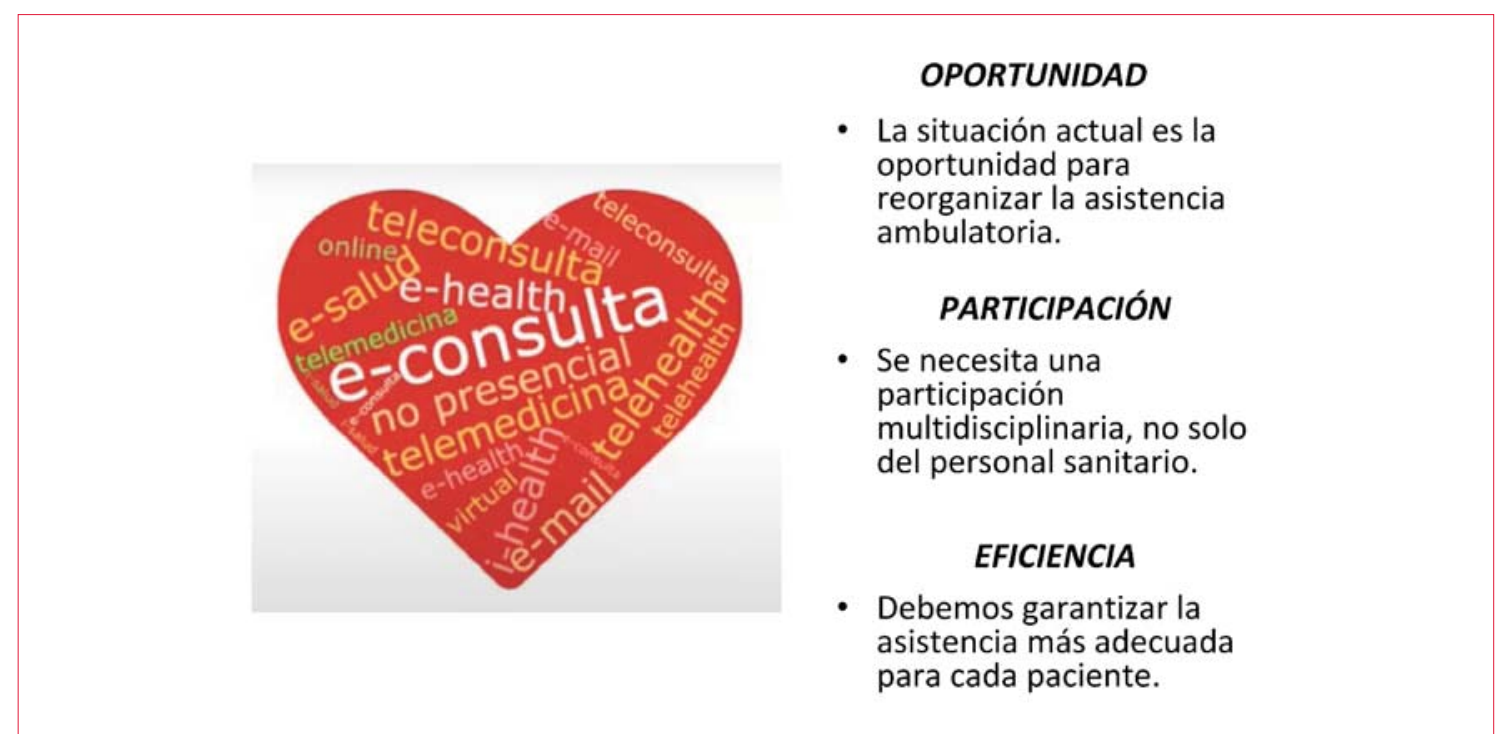

Figura 1. Modificada de presentación de la Dra. Pilar Mazón Ramos en https://secardiologia.es/multimedia/directos-online/11549-cambios-organizacion-asistencial-cardiologia-pandemia-covid-19

tipo de relación asistencial para la cual está disponible en Uruguay la tecnología necesaria, pero que no era usada, salvo excepciones. La pandemia permitió confirmar y hacer evidente, además, que un número importante de pacientes podría ser manejado a distancia sin comprometer su salud ni la calidad asistencial, que la logística necesaria para practicarla es mínima, y que fue aceptada por médicos y pacientes ${ }^{(1)}$. Incluso se ha recomendado su implementación por adelantado a una crisis y hasta fue considerada una forma ideal o perfecta para mantener la asistencia durante una epidemia ${ }^{(2,3)}$. En situaciones de excepción, como la actual, es también una alternativa para proteger al trabajador de la salud ${ }^{(4)}$.

Las modalidades de actividad asistencial no presencial han llegado para quedarse y deberían ser desarrolladas, organizadas y remuneradas de forma adecuada. Significará un cambio cultural en los pacientes, en los médicos y en los prestadores, del cual todos se beneficiarán. Redundará, finalmente, en un beneficio para la salud pública y la sociedad toda. Establecer una controversia entre la asistencia presencial como paradigma del humanismo médico y el uso de la tecnología, es un falso dilema. Ninguna modalidad sustituye a la otra, son complementarias y se potencian mutuamente.

Hace unos años, asistí a una conferencia del cardiólogo español Ignacio Fernández Lozano, que me impactó y despertó mi interés en el asunto. Dijo, palabras más, palabras menos: "La tecnología ha permitido avances increíbles en nuestras capacidades diagnósticas y terapéuticas, sin embargo, en el único ámbito en el cual los médicos seguimos haciendo lo mismo que hace 200 años, es en la consulta médi- $c a$ ". No estaba negando la necesaria vinculación humana directa del médico con su paciente, esta es siempre necesaria e imprescindible en muchísimas situaciones. Pero en pacientes crónicos conocidos, con enfermedades cardiovasculares estables, que representan un porcentaje muy importante de la consulta cardiológica clínica habitual, una gran mayoría de los controles de rutina presenciales podrían obviarse. El concurrir a una consulta, con el tiempo útil que implica, tanto para el médico como para el paciente, que para llegar a esa instancia debe sortear la burocracia que puede significar esperar meses, perder horas/días de trabajo, trasladarse (la distancia deja de ser un obstáculo para la correcta asistencia), a veces con el único objetivo de controlar la presión arterial o evaluar resultados de un examen de laboratorio, o retirar recetas, no parece imprescindible y puede ser claramente sustituida. Además, con ventajas. Según el Dr. Fernández Lozano, este tipo de consultas podría alcanzar hasta el 70\%-80\% en las policlínicas de cardiología clínica. En reciente actividad on-line de la Sociedad Española de Cardiología (SEC), la Dra. Pilar Mazón Ramos mostró datos de 2019, precoronavirus, de su hospital en Galicia. Se realizan allí anualmente más de 5.000 e-consultas (25-30 por día); en una tercera parte de las primeras consultas cardiológicas, los pacientes fueron resueltos con esta modalidad. Se trató de dudas en electrocardiograma (ECG), consultas por tratamiento, es decir, de situaciones en las que no era necesario ver al paciente; además, el tiempo medio para acceder a la e-consulta fue de tres días. (https://secardiologia.es/multimedia/ directos- online/11549-cambios-organizacion- 


\begin{tabular}{|c|c|c|c|}
\hline Dato Iime & Systolic & Diastolic & Pulso \\
\hline May 17 2020 15:22:58 & 127 & 63 & 60 \\
\hline May 17 2020 15:21:18 & 118 & 61 & 60 \\
\hline May 17 2020 15:19:38 & 121 & 57 & 58 \\
\hline May 06 2020 15:43:06 & 133 & 81 & 48 \\
\hline May 06 2020 15:41:25 & 134 & 77 & 48 \\
\hline May 06 2020 15:39:40 & 133 & 77 & 49 \\
\hline May 06 2020 15:23:24 & 129 & 69 & 49 \\
\hline \begin{tabular}{c} 
May 06 2020 15:21:42 \\
\hline May 06 2020 15:20:01
\end{tabular} & 129 & 70 & 49 \\
\hline $\begin{array}{c}\text { Apr 20 2020 } \\
15: 29: 53\end{array}$ & 130 & 73 & 53 \\
\hline $\begin{array}{c}\text { Apr 20 2020 } \\
15: 28: 11\end{array}$ & 123 & 74 & 54 \\
\hline $\begin{array}{c}\text { Apr 20 2020 } \\
15: 26: 30\end{array}$ & 126 & 72 & 55 \\
\hline
\end{tabular}

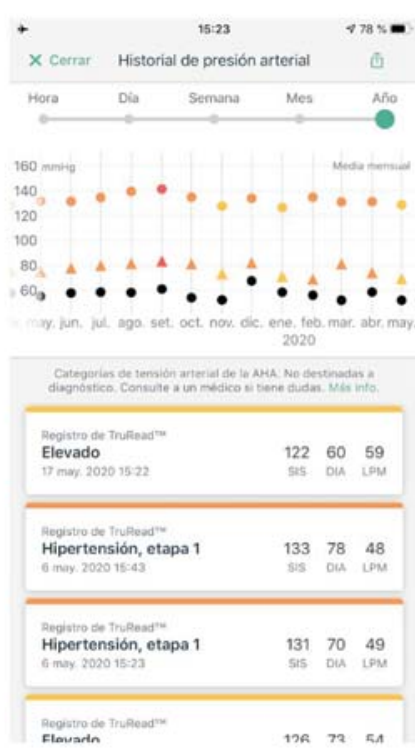

Figura 2. Aplicación de teléfono celular inteligente donde se registra el historial de presión arterial controlada por un aparato digital. A la derecha la curva de los promedios del año y a la izquierda los datos de los últimos controles. En cada control el aparato toma la presión en tres oportunidades y guarda el promedio de estas.

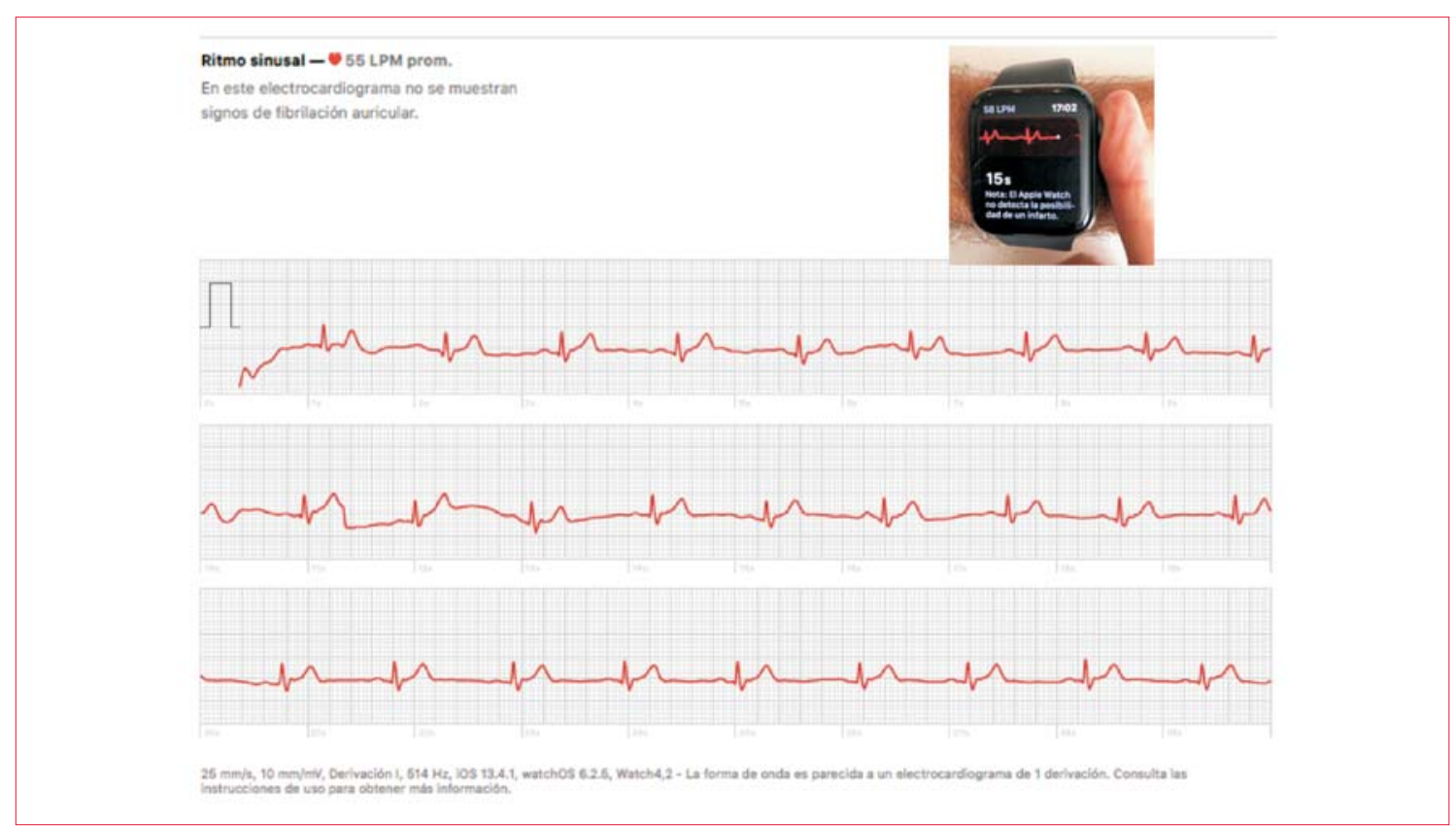

Figura 3. Electrocardiograma de una derivación obtenido con reloj iWatch 4, PDF enviado por WhatsApp.

asistencial- cardiologia-pandemia-covid-19) (figura 1).

También el uso de la tecnología enriquece la asistencia presencial. Mediante aparatos de presión digitales y aplicaciones gratuitas en los celulares, podremos tener muchas mediciones de presión arterial, además de frecuencia cardíaca, nivel de actividad del paciente, y con pocas sofisticaciones más, ya disponibles, registros periódicos del ECG, temperatura y otras muchas variables (seleccionables para cada caso), incluso de laboratorio, que nos permitirán, aun a distancia, un mejor seguimiento (figuras 2,3 y 4). La frecuencia de los controles a distancia se determinará en cada caso en particular de acuerdo a la situación clínica, psicológica y a la decisión del paciente. Por su parte, la atención presencial debe ser mantenida, mejorando su eficiencia. No debería ocurrir que el paciente asista un día a la consulta, otro a realizarse un ECG, y así con todos los estudios que se le soliciten. Lo presencial debe 

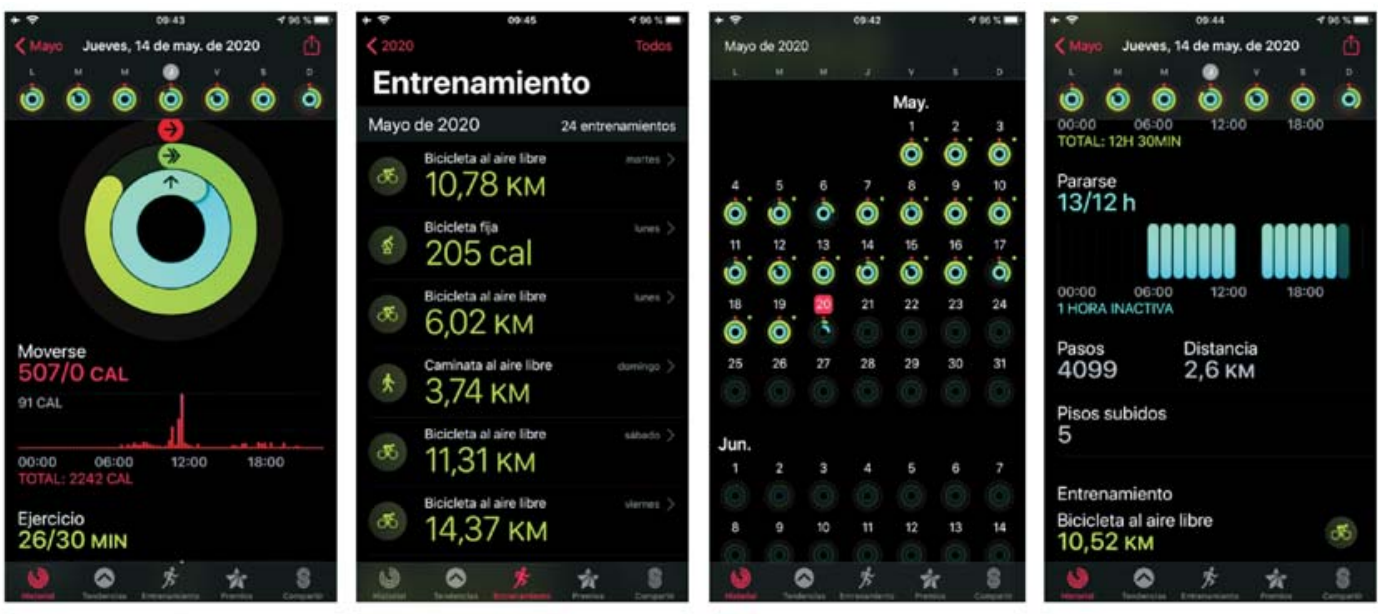

Figura 4. Pantallas de una aplicación de teléfono celular referida a la cuantificación de la actividad física.

modificarse y la situación actual nos permitió verlo claramente, lo transformó en imprescindible. Deben considerarse también otros efectos beneficiosos indirectos, como la disminución de aglomeraciones de pacientes y familiares en los centros asistenciales, y del tránsito alrededor de éstos.

\section{"Cuando antes se aparte al paciente de la influencia deprimente del hospital, más rápida será su convalecencia". Charles H. Mayo (1865-1939). Médico, cofundador de la Clínica Mayo.}

La Asociación Americana de Medicina (AMA, por su sigla en inglés) publicó recientemente on line una guía completa para la implementación de la telemedicina en la práctica clínica, cuya lectura recomendamos (https://www.ama-assn.org/practice- management/ digital/ama-quick-guide-telemedicine- practice).

También en nuestro país se han dado pasos en ese sentido. Recientemente (2/4/2020) se promulgó en el Parlamento una ley caratulada: “Aprobación de los lineamientos generales para la implementación y desarrollo de la telemedicina como prestación de los servicios de salud", (https://www.impo.com. uy/bases/leyes/19869-2020). Esta ley establece los principios en los cuales se fundamenta este tipo de práctica: universalidad, equidad, calidad, eficiencia, descentralización, complementariedad y confidencialidad.

Supone, como mencionamos antes, un cambio cultural en los pacientes, que deben entenderlo, incorporarlo y estar dispuestos a colaborar en su aprendizaje, y uso, por parte de los médicos, que deberán reciclarse y adoptar esta modalidad de trabajo como parte de su horario laboral, colaborando en desarrollarla y potenciarla educando a los pacientes, y de los prestadores que deben apoyar su instrumentación, difundirla y establecer una retribución adecuada para la tarea. Un lindo desafío para todos, de los muchos que nos dejará esta pandemia.

\section{Monitoreo remoto}

Otra modalidad asistencial con gran desarrollo en el mundo, potenciada aún más por esta crisis global, es el control a distancia (remote monitoring) de los dispositivos cardiovasculares implantables, concretamente marcapasos (MP) y cardiodesfibriladores (DAI). La efectividad de estos dispositivos es indiscutible. Pero su seguridad depende de un buen seguimiento y de este deriva el rendimiento final de la terapia. Los riesgos de su uso son bajos, pero existen (depleción inesperada de la batería, fractura de un electrodo, interrupción de un circuito de alto voltaje, falla de detección o de captura, etcétera). Por otra parte, la población portadora de dispositivos crece permanentemente. Esto lleva a una saturación de las policlínicas de control que repercute inevitablemente en su calidad. Intuitivamente el control a distancia impresiona ser superior. Supongamos, por ejemplo, a un paciente que se controla cada seis meses, uno de cuyos electrodos deja de funcionar por cualquier motivo un mes después del control. Puede presentar algún síntoma o no, y recién diagnosticaremos ese problema cinco meses más tarde. $\mathrm{O}$ el paciente presenta múltiples episodios de fibrilación auricular y requeriría modificar su tratamiento. O más evidente aún, un paciente con DAI, con un problema en el circuito de alto voltaje, que no permitirá dar un choque en caso de necesitarlo. De existir control remoto, se diagnosticaría de inmediato el problema y podría ser solucionado. Este potencial beneficio, que impresiona como muy obvio, también ha sido demostrado en ensayos clínicos. Varios estudios han puesto en evidencia no solo que el monitoreo remoto permite una solución más 


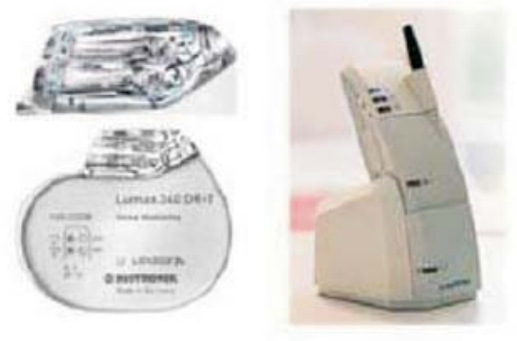

BIOTRONIK Home Monitoring?

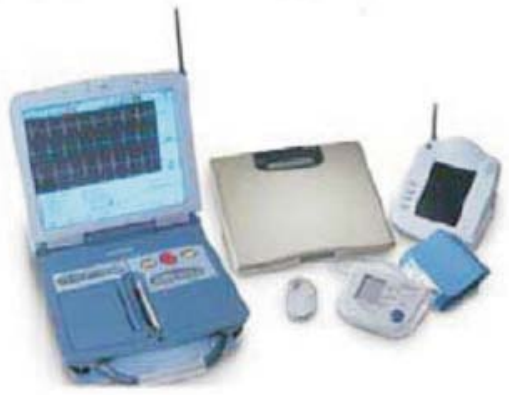

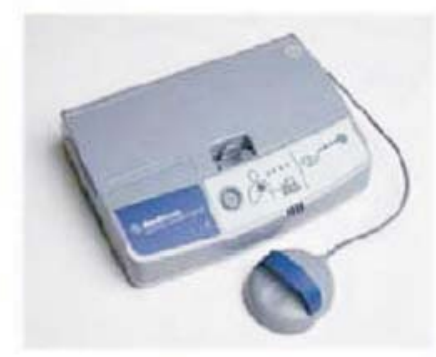

Mectronice CareLink@ Network

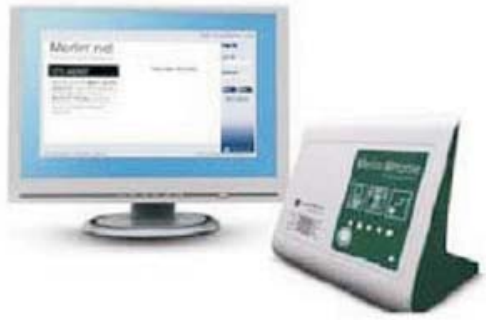

Boston Scientific Latitude@ Patient Management System

Merlin.neto Patient Care Network (PCN) St. Jude Medical

Figura 5. Distintos sistemas de monitoreo remoto de MP y DAI.

rápida de eventuales problemas ${ }^{(5-8)}$, sino que particularmente en portadores de DAI, ayuda a reducir la cantidad de choques apropiados e inapropiados (que comprometen el pronóstico además de la duración de la batería), aumentando la sobrevida de los pacientes ${ }^{(9,10)}$.

Aunque la mayoría de las grandes empresas fabricantes de dispositivos tienen su propio sistema (Abbot, Biotronik, Boston, Medtronic), referenciamos solamente los realizados con el sistema Home Monitoring $^{\circledR}$ de Biotronik ${ }^{(5,7-9)}$, o el sistema CareLink de Medtronic ${ }^{(6)}$ (figura 5).

En el Congreso anual 2020 de la Heart Rhythm Society, realizado en forma virtual, en la sesión "Ensayos clínicos que cambiarán la práctica", se presentó un estudio con un nuevo y más sencillo sistema de monitoreo remoto de MP realizado en Europa y Estados Unidos ${ }^{(11)}$. Se utilizó la tecnología BlueSync de Medtronic ${ }^{\circledR}$, mediante la cual el dispositivo se comunica automáticamente a través de bluetooth con una aplicación en el celular o tableta (MyCareLink Heart ${ }^{\text {TN }}$ App) y los datos del control son transmitidos a una red central debidamente encriptados para seguridad. Este sistema fue evaluado positivamente (95\% de transmisiones efectivas) comparado con otros tres sistemas de monitoreo remoto de dispositivos. En la presentación se destaca la importancia de lograr el empoderamiento de los pacientes, involucrándolos positivamente en el cuidado de su salud ${ }^{(11)}$ (figura 6).
Por último y jerarquizando aún más la importancia de esta tecnología, los MP y DAI actuales permiten la monitorización a distancia de otra serie de parámetros, además de los específicamente vinculados a la estimulación (nivel de fluidos, actividad del paciente, impedancia torácica) que adecuadamente procesados permiten en conjunto un seguimiento clínico estrecho, por ejemplo del paciente con insuficiencia cardíaca, y mediante software especializado establecer las alarmas y categorías de riesgo que permitan una toma de decisiones precoz previniendo descompensaciones ${ }^{(12)}$.

"No es concebible que hoy en día se implante un marcapaso que no sea posible controlar en forma remota". Dr. José Luis Zamorano. (CardioTV-SEC: Cambios en la organización asistencial en cardiología por la pandemia

COVID-19)

Todas las guías y los consensos sobre el tema recomiendan el seguimiento remoto(13). El Consenso de 2015 de la Heart Rhythm Society establece como recomendación clase I, nivel de evidencia A, "el uso de monitoreo remoto de los dispositivos además de un control anual presencial de existir la capacidad tecnológica de hacerlo, que debe ser ofrecido al paciente como parte de su seguimiento de rutina"(14). Un ensayo clínico randomizado japonés, publicado en mayo de 2020, demostró la seguridad del control remoto con un control presencial cada dos años ${ }^{(15)}$. Sin embargo, parece ser un tema no considerado 


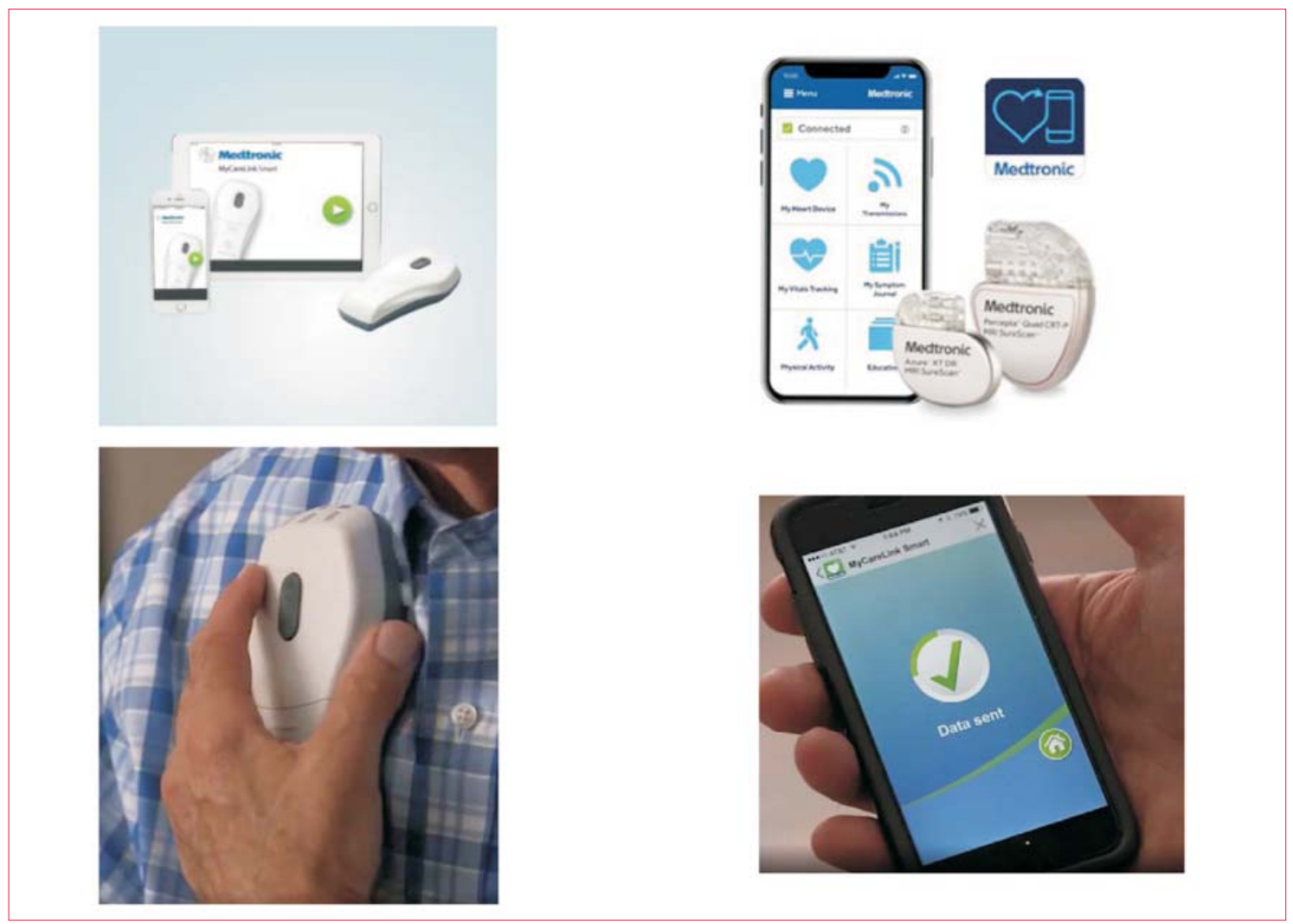

Figura 6. Sistema CareLink de Medtronic utilizando una aplicación que se instala en el celular, McOs o Android. Con un dispositivo, el paciente interroga el MP y los datos son enviados utilizando bluetooth al celular o la tableta. Desde allí se envían al servicio de MP.

por nuestro sistema asistencial, en el cual tampoco está adecuadamente jerarquizado el seguimiento presencial habitual de estos dispositivos. Está claro que la evolución hacia este tipo de controles exige de la participación de los pacientes que deben conocerlo, aceptarlo y recibir una devolución cada vez que son controlados, de los médicos y servicios que deberán organizar la infraestructura, y de los prestadores y el sistema de salud para invertir en su ejecución, pues tendrá efectos beneficiosos en los pacientes y en los costos ${ }^{(16)}$. Sin olvidar las ventajas de disponer por defecto de una base de datos con la información completa del funcionamiento del dispositivo de cada paciente y los demás beneficios adicionales ya referidos para el caso de la telemedicina (descongestionar centros de salud, eliminación de la distancia como barrera). También las empresas fabricantes deben colaborar, facilitando la instalación de la tecnología necesaria, y sería de enorme utilidad que unificaran en el futuro sus plataformas. En países desarrollados se llega a controlar más de $80 \%$ de los pacientes con esta modalidad, existiendo personal dedicado (usualmente técnicos o enfermeros específicamente entrenados) que consulta a los médicos de ser necesario y hace las devoluciones correspondientes.
Vinculado también al control de pacientes con trastornos del ritmo cardíaco, existen disponibles diversas modalidades de dispositivos o relojes que permiten registrar el ECG, hasta de 12 derivaciones, y enviar el registro de manera muy sencilla a través de un teléfono celular. En Uruguay hay otros sistemas en desarrollo que transmiten el ECG de hasta 6 derivaciones en forma permanente a través de la web, pudiendo ser visto/revisado accediendo a distancia. Esta tecnología, existente y en desarrollo, facilitará la monitorización ambulatoria del ritmo cardíaco y mejorará el manejo correcto de los pacientes, con inversiones menores ${ }^{(17)}$ (figura 7).

\section{El derecho a la privacidad, la salud y el} empoderamiento de los ciudadanos

Desde el año pasado, Uruguay inició un camino para que cada ciudadano tenga una historia clínica electrónica a la cual podrá acceder, como también lo harán los profesionales de la salud que lo asistan en cualquier lugar del territorio. Será un complemento imprescindible que optimizará la asistencia a distancia y significará, como lo refiere el Dr. Baltasar Aguilar en su excelente artículo recientemente publicado en esta revista(18), un cambio de 


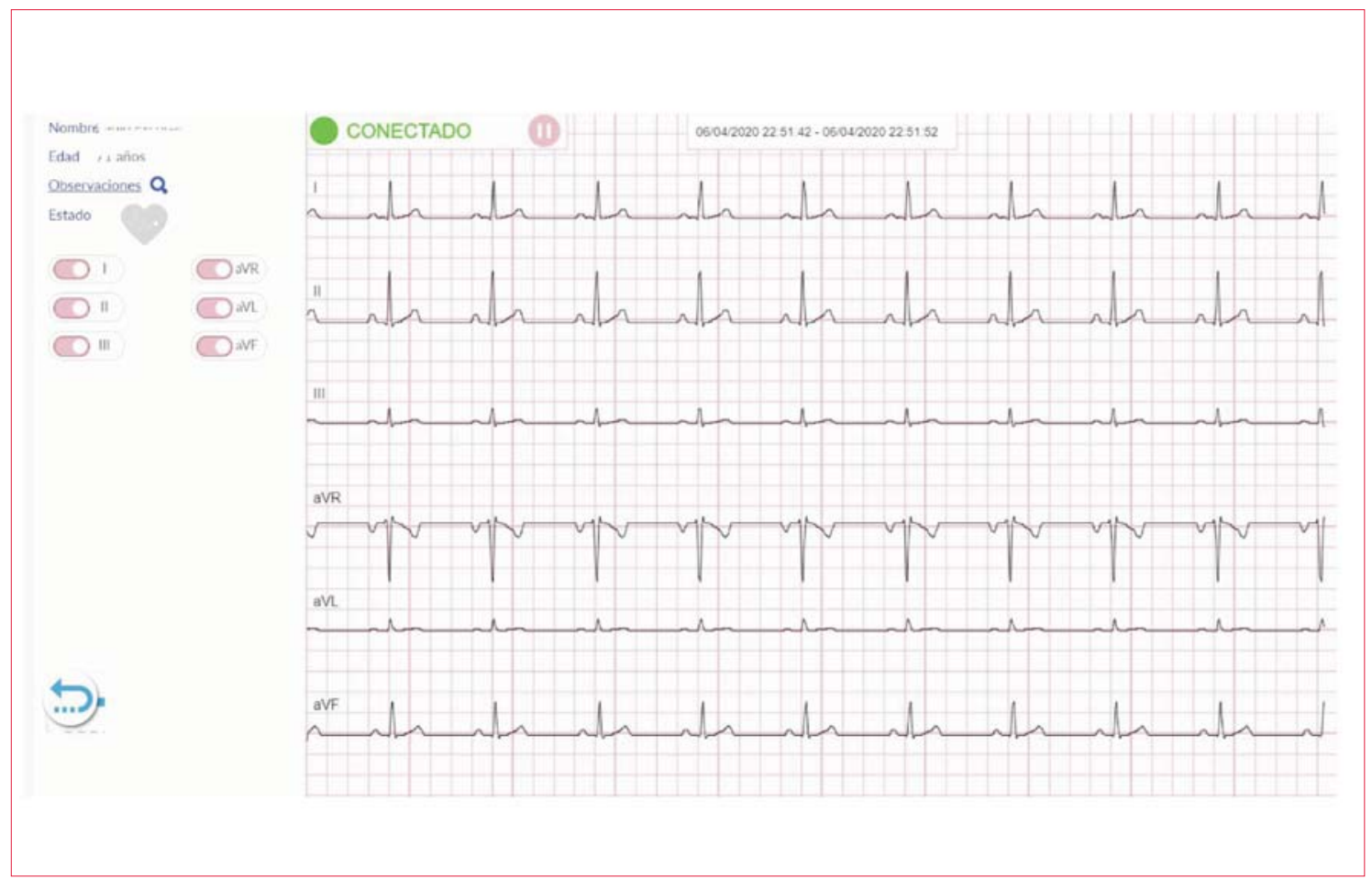

Figura 7. Imagen del registro permanente de seis derivaciones obtenido on line, con sistema de registro Galeno-Sys, en desarrollo en Uruguay.

paradigma, un cambio cultural. Como él lo plantea, desde el mismo momento que se hizo público el tema, han surgido polémicas y cuestionamientos, fundamentalmente vinculados a la privacidad y la seguridad de los datos, el derecho a la intimidad, las potenciales "filtraciones" o usos comerciales de los datos, etcétera. Las precauciones han sido consideradas en la reglamentación correspondiente, los mecanismos de protección se han extremado para poder desarrollar con la mayor de las seguridades posibles este proceso que, coincidimos con el Dr. Aguilar, es inevitable.

El dilema entre privacidad/derechos versus salud de las personas frecuentemente es presentado como una limitación para el uso de la tecnología en la atención sanitaria. Citamos al respecto al historiador Yuval Noah Harari, quien en reciente y conocida entrevista vinculada a la pandemia de coronavirus ${ }^{(19)}$, lo refiere como "un razonamiento falaz. En el hecho de pedir a la gente que elija entre intimidad y salud reside en realidad la raíz misma del problema. Porque se trata de una falsa elección. Podemos y debemos disfrutar tanto de la intimidad como de la salud. Es posible proteger nuestra salud y detener la epidemia de coronavirus sin tener que instituir regímenes de vigilancia totalitarios, sino más bien empoderando a los ciudadanos".

Pasada esta epidemia, el inevitable desarrollo de la telemedicina y el empoderamiento de los ciu- dadanos serán procesos que deberán caminar juntos.

$$
\begin{array}{r}
\text { "Me interesa el futuro pues es el lugar donde voy a } \\
\text { pasar el resto de mi vida". } \\
\text { Woody Allen }
\end{array}
$$

\section{Oportunidad}

Que las crisis son también momentos de oportunidades, es una idea generalmente aceptada. La actual pandemia introdujo una pausa en el ritmo vertiginoso de la sociedad moderna y ha generado una toma de conciencia global y cuestionamientos sobre muchos aspectos de nuestra vida, tanto en lo personal como en lo social. No desperdiciemos esta pausa. Parece ser una gran oportunidad para implementar cambios que coloquen a la salud humana, sus sistemas sanitarios, sus trabajadores y la educación en salud de la comunidad, en el lugar de jerarquía que deben tener en un mundo que soñamos como más saludable.

Con un objetivo más modesto, y vinculado a nuestra especialidad, tenemos muchas expectativas en la oportunidad que se presenta para el sistema sanitario de nuestro país, en el desarrollo de la telemedicina y del monitoreo remoto en todas sus variedades. Son modalidades para cuya implementación existe la tecnología disponible y que mejoran sustancialmente la calidad asistencial y la eficiencia en el uso de los recursos.

Ojalá seamos capaces de aprovecharla. 
Walter Reyes Caorsi,

https://orcid.org/0000-0002-4670-5765

Este artículo fue aceptado para su publicación por: Editor jefe Dr. Gerardo Soca.

\section{Bibliografía}

1. Bashshur R, Doarn CR, Frenk JM, Kvedar JC, Woolliscroft JO. Telemedicine and the COVID-19 Pandemic, Lessons for the future. Telemed J E Health 2020;26(5):571-3. doi: 10.1089/tmj.2020. 29040.rb

2. Mann DM, Chen J, Chunara R, Testa PA, Nov O. Covid-19 transforms health care through telemedicine: evidence from the field. J Am Med Inform Assoc. 2020. 23. pii: ocaa072. doi: 10.1093/jamia/ ocaa072. [online ahead of print]

3. Hollander JE, Carr BG. Virtually perfect? Telemedicine for Covid-19. N Engl J Med. 2020;382(18): 1679-81. doi: 10.1056/NEJMp2003539

4. Moazzami B, Razavi-Khorasani N, Moghadam AD, Farokhi E, Rezaei N. Covid-19 and Telemedicine: immediate action required for maintaining health care providers well-being. J Clin Virol. 2020;126.104345.doi: 10.1016/j.jcv.2020.104345

5. Mabo P, Victor F, Bazin P, Ahres S, Babuty D, Da Costa A, et al. A randomized trial of long-term remote monitoring of pacemaker recipients. (The COMPAS Trial). Eur Heart J. 2012; 33(9): 1105-11. doi:10.1093/eurheartj/ehr419

6. Crossley GH, Boyle A, Vitense H, Chang Y, Mead RH; for the CONNECT Investigators. The CONNECT (Clinical evaluation of remote notification to reduce time to clinical decision) trial. J Am Coll Cardiol. 2011; 57: 1181-9. doi:10.1016/j.jacc. 2010.12 .012

7. Varma N, Epstein AE, Irimpen A, Schweikert R, Love C; for the TRUST Investigators. Efficacy and safety of automatic remote monitoring for implantable cardioverter-defibrillator follow-up. The Lumos T safely reduces routine office follow-up (TRUST) Trial. Circulation 2010;122(4);325-32. doi: 10.1161/CIRCULATIONAHA.110.937409

8. Guédon-Moreau L, Locroix D, Sadoul N, Clémenty J, Kouakam C, Hermida JS, et al. A randomized study of remote follow-up of implantable cardioverter defibrillator: safety and efficacy report of the ECOST trial. Eur Heart J. 2013; 34(8): 605-14 doi:10.1093/eurheartj/ehs425

9. Guédon-Moreau L, Kouakam C, Klug D, Marquié $\mathbf{C}$, Brigadeau F, Boulè $\mathbf{S}$, et al. Decreased Delivery of Inappropriate Shocks Achieved by Remote Monitoring of ICD: a substudy of the ECOST trial.
J Cardiovasc Electrophysiol. 2014; 25(7): 763-70. doi: 10.1111/jce. 12405

10. Saxon LA, Hayes DL, Guilliam FR, Heidenreich PA, Day J, Seth M, et al. Long-term outcome after CRT and ICD implantation and influence of device remote follow-up. The ALTITUDE survival study. Circulation 2010; 122 (23): 2359-67. doi: 10.1161/CIRCULATIONAHA.110.960633

11. Tarakji KG, Zafidi AM, Zwelbel SL, Seller A, Roberts PR, Shaik NA, et al. Performance of first in the world pacemaker to use smart device app for remote monitoring. Heart Rhythm Society Annual Meeting 2020 (virtual); 2020 [Consulta: 23 May 2020]. Disponible en: https://www.hrsonline.org/ HRS2020Science

12. Ahmed FZ, Taylor JK, Green C, Moore L, Goo$\operatorname{de~A,~Black~P,~et~al.~Triage-HF~plus:~a~novel~devi-~}$ ce-based remote monitoring pathway to identify worsening heart failure. ESC Heart Fail. 2020;7(1): 107-16. doi: 10.1002/ehf2.12529

13. Brignole M, Auricchio A, Barón-Esquivias G, Bordachar P, Boriani G, Breithardt OA, et al. 2013 ESC Guidelines on Cardiac Pacing and Cardiac Resynchronization Therapy. European Heart Journal 2013, 34(29): 2281-329. doi: 10.1093/eurheartj/eht150

14. Slotwiner D, Varma N, Akar JG, Annas G, Beardsall M, Fogel RI, et al. HRS expert consensus statement on remote interrogation and monitoring for cardiovascular implantable electronic devices. Heart Rhythm J 2015; 12(7): e69-100. doi: 10.1016/j.hrthm.2015.05.008

15. Watanabe E, Yamazaki F, Goto T, Asal T, Yamamoto T, Hirooka K, et al. Remote management of pacemaker patients with biennial In-clinic evaluation. Continuous home monitoring in the Japanese at home study: a randomized clinical trial. Circ Arrhythm Electrophysiol. 2020;13: e007734. doi: 10.1161/CIRCEP.119.007734

16. Merzegalli M, Lunati M, Landolina M, Perego GB, Ricci RP, Guenzati G, et al. Remote monitoring of CRT-ICD: the multicenter Italian CareLink evaluation-Ease of use, acceptance, and organizational implications. Pacing Clin Electrophysiol. 2008; 31(10): 1259-64. doi: 10.1111/j.1540-8159.2008.01175.x

17. Galeno_Sys [Internet]. Montevideo: ThalesLab; 2020 [consulta 27 May 2020]. Disponible en: www. dataflow.com.uy/Galeno1.html

18. Aguilar Fleitas B. La Medicina, ien el enjambre?. Rev Urug Cardiol. 2020;35(1): 8-11 doi: 10.29277/ cardio.35.1.3

19. Harari YH. The world after coronavirus [Internet]. 2020 [Consulta 27 May 2020]. Disponible en: https://www.ft.com/content/19d90308-6858-11eaa3c9-1fe6fedcca75 